\title{
OCCULTATION OF RADIO SOURCES FOR THE LINKAGE OF RADIO AND STELLAR REFERENCE FRAMES
}

\author{
Mitsuru Sôma, Masanori MiYamoto and Shinko AokI \\ National Astronomical Observatory \\ Mitaka, Tokyo 181, Japan
}

\begin{abstract}
The right ascension of the radio source $3 \mathrm{C} 273 \mathrm{~B}$, which serves as a right ascension zero point in radio astrometric work, has been determined from lunar occultations and photographic observations.

We re-analyze here the lunar occultations of $3 \mathrm{C} 273 \mathrm{~B}$ using the recent precise lunar ephemeris and obtain its right ascension referred to the FK5 equinox at J2000.0. The obtained right ascension is $12^{\mathrm{h}} 29^{\mathrm{m}} 06^{\mathrm{s}} 6946 \pm 0.007$ at its mean observation epoch of 1963.62 .

Predictions of occultations of radio sources by the Moon and planets are also given. Observations of them are encouraged in order to improve the accuracy of the linkage between radio and stellar reference frames.
\end{abstract}

\section{Introduction}

In the astrometric observations of radio sources by VLBI, relative right ascensions can only be determined. The radio source $3 \mathrm{C} 273 \mathrm{~B}$ has been recommended as right ascension zero point in radio astrometric work and its right ascension $12^{\mathrm{h}} 26^{\mathrm{m}} 33^{\mathrm{s}} 246$ referred to the FK4 equinox at B1950.0 was based on lunar occultations and photographic observations (Hazard et al., 1971). The occultations were analyzed using the lunar ephemeris $j=2$ based on the theory by Brown with the corrections to the ephemeris, which were deduced, with slight modifications, from an analysis of occultations of stars by Morrison and Sadler (1969).

There is a confusion about this adopted right ascension. For example, Witzel and Jonston (1982) assume that in this value the E-terms of aberration are included while Clark et al. (1976) and Elsmore (1979) assume that the terms are not included. The right ascension $12^{\mathrm{h}} 29^{\mathrm{m}} 06^{\mathrm{s}} 6997$ referred to the FK5 equinox at J2000.0 was converted from the B1950.0 value, but its conversion procedure leaves some problem as Aoki et al. (1986) pointed out.

In Sect. 2 we re-analyze the lunar occultations of $3 \mathrm{C} 273 \mathrm{~B}$ used by Hazard et al. using the recent precise lunar ephemeris. In order to improve the accuracy of the linkage between radio and stellar reference frames, observations of occultations of radio sources by the Moon and planets are important. In Sect. 3 prediction of occultations by the Moon is given and in Sect. 4 prediction of occultations by the planets is given. 


\section{Re-analysis of occultations}

We analyze the lunar occultations of 3C273B used by Hazard et al. (1971), based on the lunar theory ELP2000-82 by Chapront-Touzé and Chapront (1982). The constants for the lunar theory and the correction to the Watts' (1963) charts we use are those obtained by Sôma (1985) from an analysis of lunar occultations of stars.

Fig. 1 shows the difference between the ephemerides used by Hazard et al. and used by us. The difference reaches about 0.3 at 1964. Fig. 2 shows the difference between the ephemeris LE200 of JPL and the ephemeris used by us. The LE200 is the lunar ephemeris appearing in The Astronomical Almanac for the year 1984 onwards and it is consistent with the planetary ephemeris DE200. The constant difference in longitude of about $0 . " 4$ shown in Fig. 2 is almost due to the difference between the center of gravity and the center of the mean limb adopted by Watts (1963). The linear trend of about 0."004/year is due to the difference in the mean motion, which probably includes the difference in the adopted precession constant. The small periodic terms are due to the difference between the adopted values for the eccentricity, inclination, etc. The difference is less than 0. " 04 .

In deriving the right ascension of $3 \mathrm{C} 273 \mathrm{~B}$, we adopt the declination $\delta=+2^{\circ} 03^{\prime} 08^{\prime \prime} .59$ (J2000.0) obtained from VLBI observations. Of 5 occultations Hazard et al. cited we omit that of 1963 Mar. 11 (grazing occultation) because the accuracy of the Watts' charts of that field is poor and the accuracy of determining the right ascension is not good due to the position angle being near $90^{\circ}$. We also omit the occultation of 1964 Sept. 7 as Hazard et al. did because of its poor observational accuracy.

The geodetic coordinates (longitude $\lambda$, latitude $\phi$, and height $h$ ) of the observatories (Australian National Radio Astronomical Observatory at Parkes and Arecibo Observatory) are taken from The Astronomical Almanac for the year 1981 (p. J46 and p. J36). The data for reducing the regional geodetic coordinates to the geocentric coordinates are taken from The Astronomical Almanac for the year 1990 (p. K13).

The obtained right ascension of 3C273B referred to the FK5 equinox at J2000.0 (without the E-terms of aberration) is

$$
\alpha=12^{\mathrm{h}} 29^{\mathrm{m}} 06^{\mathrm{s}} \cdot 6946 \pm 00^{\mathrm{s}} .007
$$

and its mean observational epoch is 1963.62 . The difference $0 . " 08$ between this value and the presently adopted value is within the accuracy of observation.

It should be noted that the value obtained here is dependent on the precession constant used in the analysis. The precession constant we use is the constant in the IAU (1976) System of Astronomical Constants (Duncombe et al., 1977).

In order to improve the accuracy of the connection between the radio reference frame and the optical reference frame, further observations of occultations of radio sources by the Moon and planets are recommended.

\section{Prediction of occultations by the Moon}

Table 1 shows the radio observatories for which lunar occultations are calculated. Coordinates of these stations are taken from The Astronomical Almanac for the year 1990 (pp. 


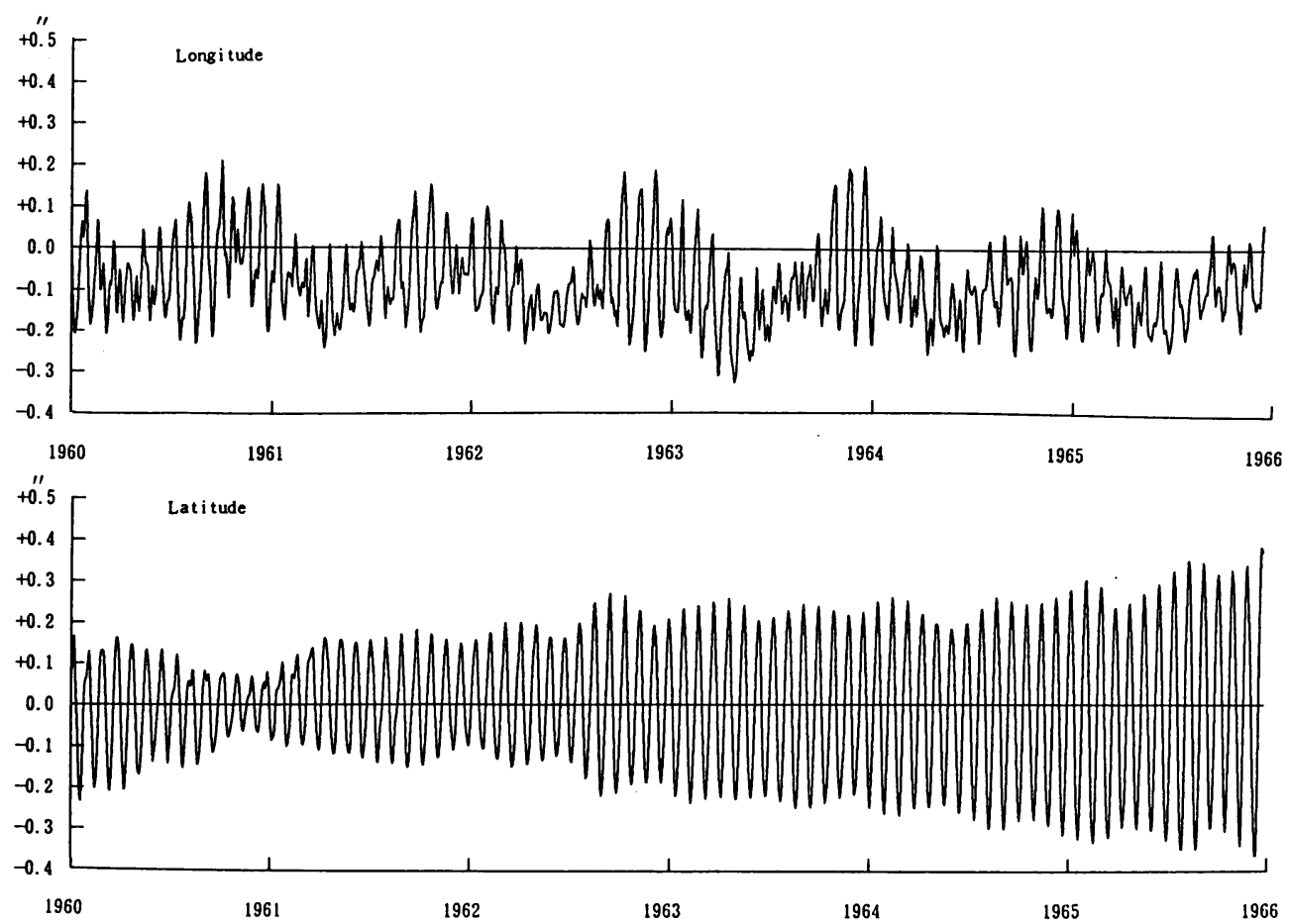

Fig. 1 Difference in lunar ephemeris (Hazard et al. minus ELP2000)
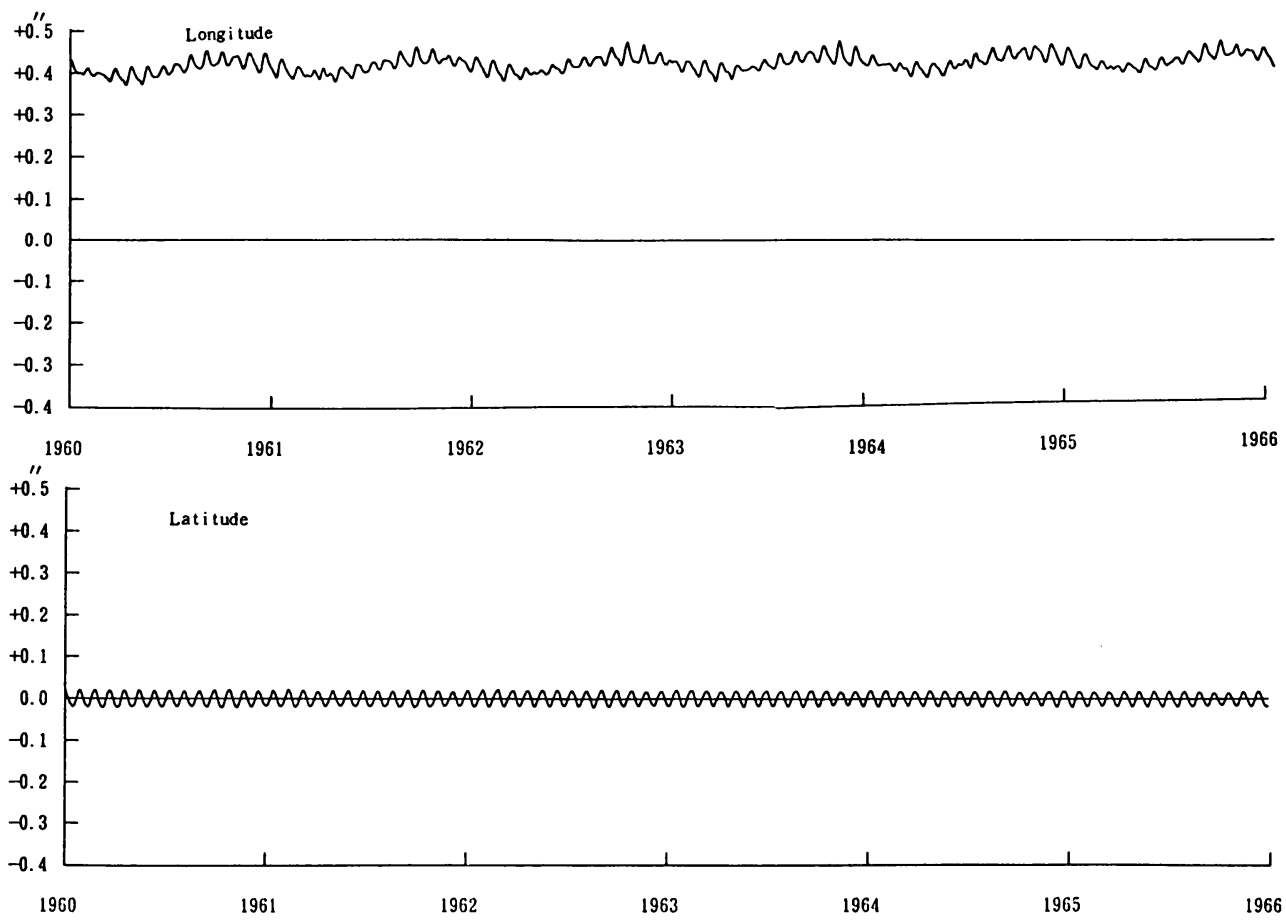

Fig. 2 Difference in lunar ephemeris (ELP2000 minus LE200) 
J6-J16). Radio sources are taken from Argue et al. (1984), Morabito et al. (1986) and the recent JPL catalog JPL 1989-3 (Sovers, 1989).

Catalogs of stars may include systematic errors of about 0.1 . Accordingly if we rely on only one radio source $3 \mathrm{C} 273 \mathrm{~B}$ for the connection between radio and stellar reference frames, the radio reference frame should include the error of that field in the stellar reference frame. Therefore it is important that positions of many radio souces should be determined independently.

Prediction of occultations by the Moon for the year 1991 is shown in Table 2. It shows the observatories where occultation is visible at more than $20^{\circ}$ above horizen when the Sun's altitude is less than $-10^{\circ}$.

Occultation of the radio source 3C273B will occur during 2000-2002.

We can provide occultation predictions for any observatory on request.

\section{Prediction of occultations by planets}

We have found 4 occultations of radio sources by planets during 1990-2000: the occultation of 1748-253 by Venus on 1992 Nov. 11, the occultation of P 2208-137 by Mercury on 1996 Mar. 7, the occultation of $P$ 2008-159 by Venus on 1998 Mar. 6, and the occultation of 1215-002 by Mercury on 1998 Sept. 26. Fig. 3 is the maps showing the regions where these occultations are visible. On the maps the sunrise and/or sunset terminator is shown, with hatches indicating the side of nighttime visibility. The radio source and the occulting planet are in the zenith for an observer at a site indicated by the center of the circular projection of the Earth; the objects are on the horizen for sites at the edge of the circle. The altitude above the horizen can be estimated for any site shown on the map; the cosine of the altitude is the distance of the site from the center of the circle divided by the radius of the circle.

Recently we received a paper about the prediction of occultations by planets written by Linfield (1989) of JPL. Our prediction is in good agreement with his prediction. In addition to the occultations we found, he predicts many possible occultations of radio sources with poor positional accuracy. Observations of occultations should be encouraged. 
Table 1. Radio Observatories

Name of Observatory

Ch: Chatanika Incoher. Scatter Fac.

HC: Hat Creek Radio Ast. Obs.

Gs: Goldstone Complex

Hv: Harvard Radio Ast. Sta.

Ri: Richmond POLARIS Obs.

Hs: Haystack Obs.

CT: Cerro Tololo Inter-Amer. Obs.

Rb: Robledo Deep Space Sta.

On: Onsala Space Obs.

SR: Radio Space Research Sta.

By: Byurakan Astrophysical Obs.

Gu: Guaribidanur Radio Obs.

Bj: Beijing Normal Univ. Obs.

Ka: Kashima Space Commun. Center

Ti: Tidbinbilla Deep Space Sta.
East Long. North Lat. Height

$\begin{array}{rrr}-147^{\circ} 27^{\prime} .1 & +65^{\circ} 06^{\prime} .2 & 235 \\ -12128.4 & +4049.1 & 1043 \\ -11650.9 & +3523.4 & 1036 \\ -10356.7 & +3038.2 & 1603 \\ -8023.1 & +2536.8 & 11 \\ -7129.3 & +4237.4 & 146 \\ -7048.9 & -3009.9 & 2215 \\ -414.9 & +4025.8 & 774 \\ +1155.2 & +5723.6 & 5 \\ +2741.2 & -2553.4 & 1382 \\ +4417.5 & +4020.1 & 1500 \\ +7726.1 & +1336.2 & - \\ +11621.6 & +3957.4 & 70 \\ +14039.8 & +3557.3 & 32 \\ +14858.8 & -3524.1 & 656\end{array}$

Table 2 Occultations by the Moon in 1991

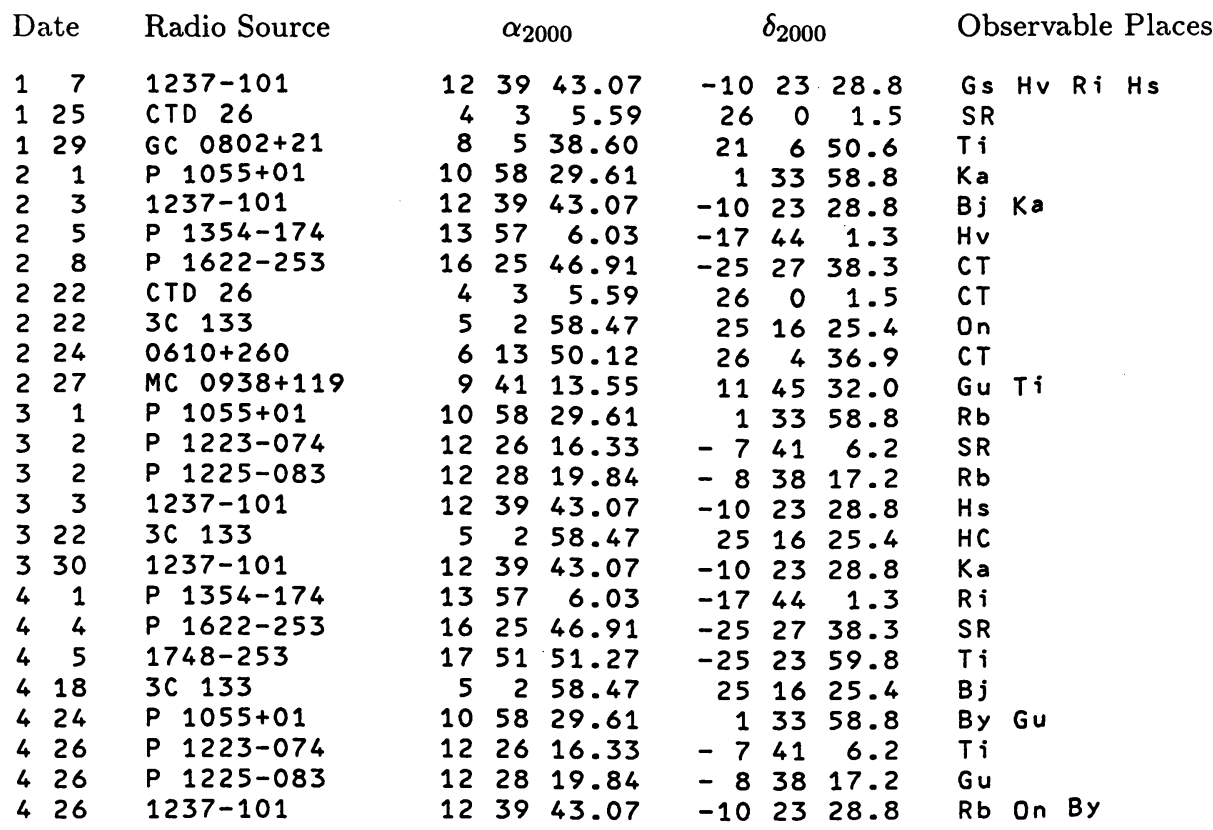


Table 2 Occultations by the Moon in 1991 (Continued)

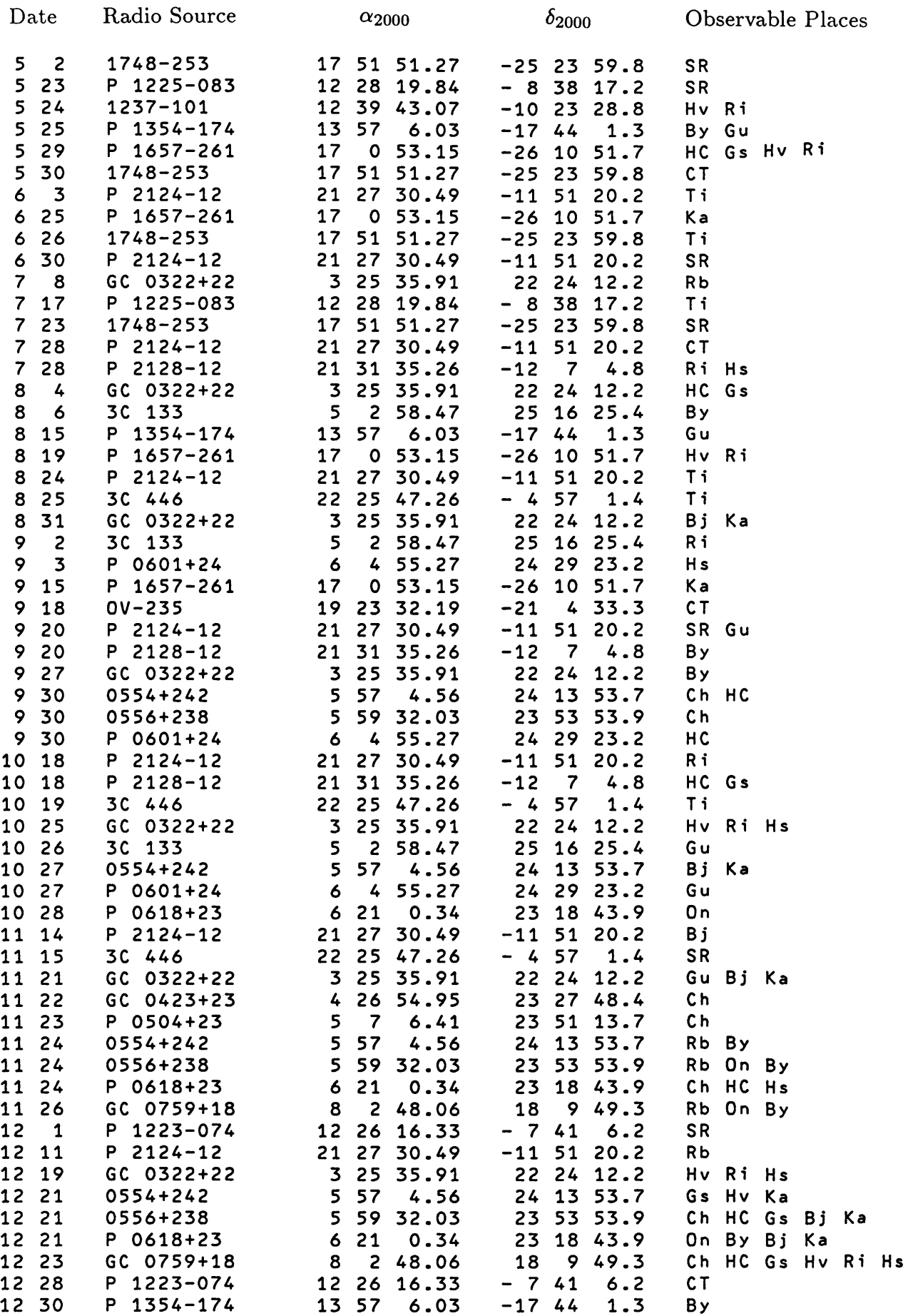




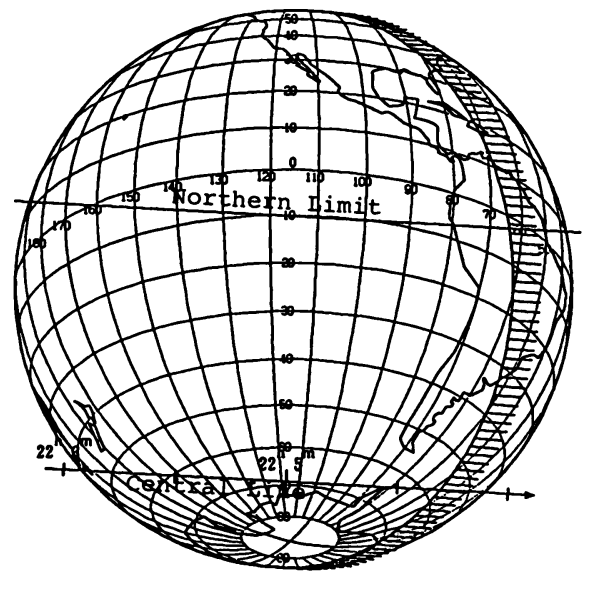

1748-253 by Venus (1992 Nov. 11)

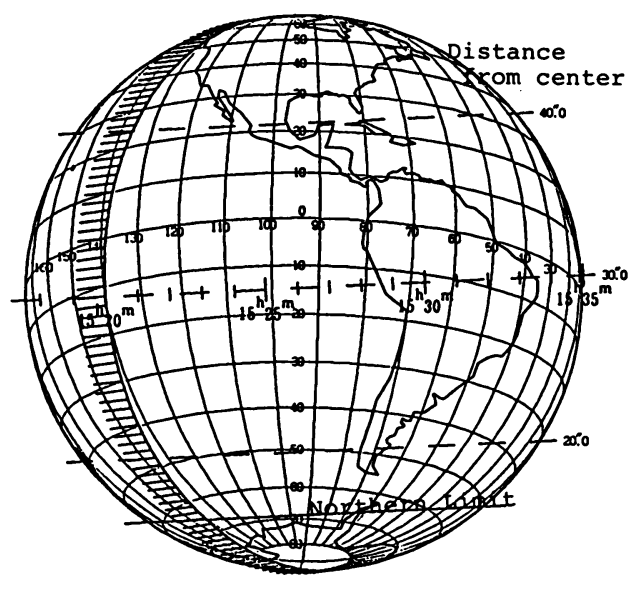

P 2008-159 by Mercury (1998 Mar. 6)

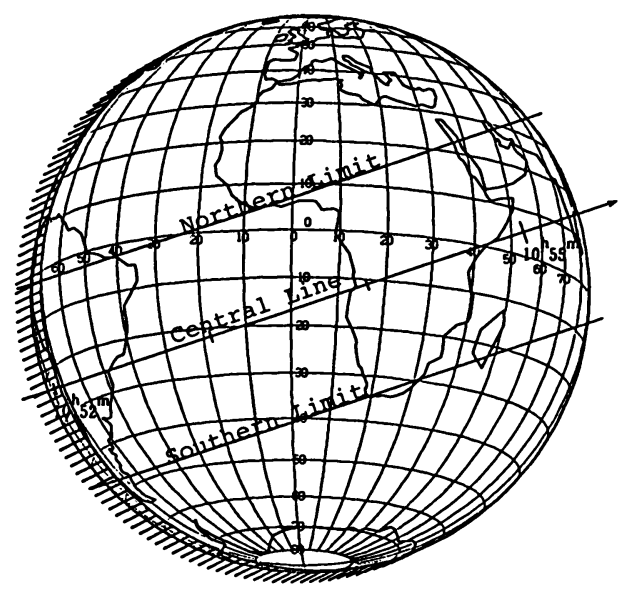

P 2208-137 by Mercury (1996 Mar. 7)

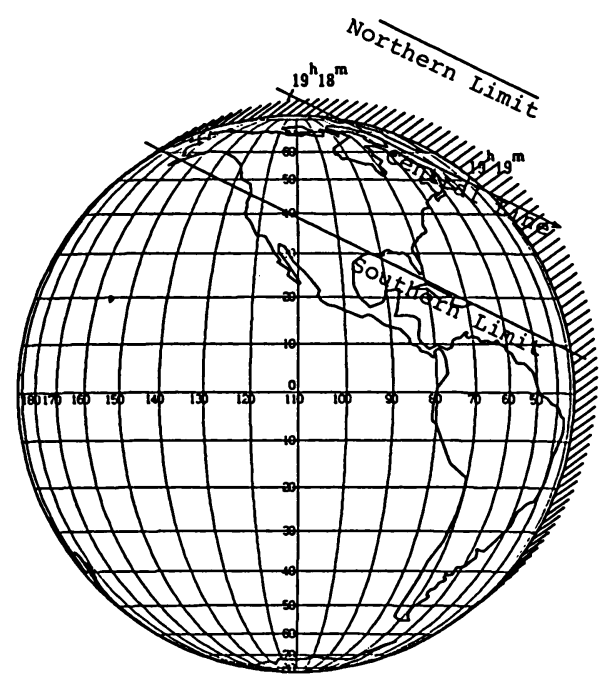

1215-002 by Mercury (1998 Sept. 26)

Fig. 3 Occultations by planets 


\section{References}

Aoki, S., Sôma, M., Nakajima, K., Niimi, Y., Fujishita, M., and Takahashi, Y. (1986) 'The conversion from the B1950 FK4-based position to the J2000 position of celestial objects', in H. K. Eichhorn and R. J. Leacock (eds.), Astrometric Techniques (IAU Symp. No 109), D. Reidel Publishing Company, Dordrecht, pp. 123-131.

Argue, A. N., de Vegt, C., Elsmore, B., Fanselow, J., Harrington, R., Hemenway, P., Johnston, K. J., Kühr, H., Kumkova, I., Niell, A. E., Walter, H., and Witzel, A. (1984) 'A catalog of selected compact radio sources for the construction of an extragalactic radio/optical reference frame', Astron. Astrophys. 130, 191-199.

Chapront-Touzé, M. and Chapront, J. (1982) 'ELP2000-82', Magnetic Tape.

Clark, T. A., Hutton, L. K., Marandino, G. E., Counselman, C. C., Robertson, D. S., Shapiro, I. I., Wittels, J. J., Hinteregger, H. F., Knight, C. A., Rogers, A. E. E., Whitney, A. R., Niell, A. E., Rönnäng, B. O., and Rydbeck, O. E. H. (1976) 'Radio source positions from very-long-baseline interferometry observations', Astron. J. 81, 599-603.

Duncombe, R. L., Fricke, W., Seidelmann, P. K., and Wilkins, G. A. (1977) 'Joint report of the working groups of IAU commission 4 on precession, planetary ephemerides, units and time-scales', Trans. IAU XVI B, 56-67.

Elsmore, B. (1979) 'Review of radio astrometry I. Radio measurements', in F. V. Prochaska and R. H. Tucker (eds.), Modern Astronomy (IAU Coll. No. 48), Univ. Obs. Vienna, pp.93-99.

Hazard, C., Sutton, J., Argue, A. N., Kenworthy, C. M., Morrison, L. V., and Murray, C. A. (1971) 'Accurate radio and optical positions of 3C273B', Nature Physical Science 233, 89-91.

Linfield, R. P. (1989) 'Using planetary occultations of radio sources for frame-tie measurements I. Motivation and search for events', draft for JPL TDA Progress Reports.

Morabito, D. D., Niell, A. E., Preston, R. A., Linfield, R. P., Wehrle, A. E., and Faulkner, J. (1986) 'VLBI observations of 416 extragalactic radio sources', Astron. J. 91, 1038-1050.

Morrison, L. V. and Sadler, Flora McBain (1969) 'An analysis of lunar occultations 1960-66', Mon. Not. Roy. Astron. Soc. 144, 129-141.

Sôma, M. (1985) 'An analysis of lunar occultations in the years 1955-1980 using the new lunar ephemeris ELP2000', Celes. Mech. 35, 45-88.

Sovers, O. J. (1989) 'JPL 1989-3', submitted to the IERS.

Watts, C. B. (1963) 'The marginal zone of the Moon', Astron. Pap. Amer. Ephem. XVII, U. S. Government Printing Office, Washington, D. C.

Witzel, A. and Johnston, K. J. (1982) 'Candidate radio sources for a radio/optical reference catalog', Abhandlungen aus der Hamburger Sternwarte Band X, Heft 3, 151-164. 


\section{Discussion}

FEISSEL: Radio source 3C273B has played a historical role in the initial definition of the right ascension origin of the kinematic reference frame, based on extragalactic objects, in agreement with that of the dynamical one based on solar system objects. In recent years, other processes have been used, e.g. (a) define the agreement with the FK5 system by a few tens of quasars whose positions are known in the FK5 system, or (b) maintain the directions of axes of the reference frame by a no-rotation condition based on well measured extragalactic radio sources.

SômA: I think occultations are still useful for checking the agreement between the radio and stellar or dynamical reference frames.

STEINERT: Radio source $3 \mathrm{C} 273$ has a variable structure. Is that not a disadvantage for a zero-point of the RA system?

SômA: Yes. I think a more stable radio source should play the role of the zero-point of right ascension.

FEISSEL: This zero-point of the radio system in RA (i.e. 3C273) has its history and it seems not necessary to change it.

Sôma: The variability of the structure, for example, has not yet been established.

MORRISON: I was involved in the original reduction of the lunar occultations which led to the position published in the paper by Hazard et al. It seems to me somewhat fortuitous that your new position reduced with a modern lunar ephemeris should agree so closely with the original value. Do you agree?

Sôma: Yes. The close agreement is merely fortuitous. In the present work, I also wanted to stress this point.

TREUHAFT: Is the dominant error contributing to your 7 millisecond error in the right ascension of $3 \mathrm{C} 273$ due to the uncertainty in the structure of the limb of the Moon?

Sôma: Yes. I think observational timing error and the error in the limb correction are the main sources of error in the derived right ascension. 